\title{
Neural tube defect diagnosis and outcomes at a tertiary South African hospital with intensive case ascertainment
}

\author{
E I Krzesinski, ${ }^{1} \mathrm{MB}$ ChB, MMed (Med Genetics), FRACP; L Geerts, ${ }^{2}$ MB ChB, MRCOG, Dip Fetal Med (UK); \\ M F Urban, ${ }^{1}$ MB BCh, FCPaed (SA), Cert Med Genetics (SA), PhD \\ ${ }^{1}$ Division of Molecular Biology and Human Genetics, Faculty of Medicine and Health Sciences, Tygerberg Academic Hospital and \\ Stellenbosch University, Cape Town, South Africa \\ ${ }^{2}$ Department of Obstetrics and Gynaecology, Faculty of Medicine and Health Sciences, Tygerberg Academic Hospital and Stellenbosch University, \\ Cape Town, South Africa
}

Corresponding author: M F Urban (urban@sun.ac.za)

Background. Neural tube defects (NTDs) are an important category of birth defect, but surveillance remains inadequate in South Africa. Objectives. To assess the identification of NTDs at a tertiary hospital using a range of prenatal, perinatal and postnatal data sources, and to estimate the impact of prenatal diagnosis and birth prevalence for the referral area.

Methods. Cases of anencephaly, encephalocele and spina bifida (SB) in a 6-year period were retrospectively identified from 5 data sources covering prenatal, perinatal and postnatal care. These were cross-correlated to avoid duplicate entries and to determine the contribution of different data sources. Details of prenatal diagnosis and termination of pregnancy (TOP) were obtained for 10 years, and birth prevalence over 2 years.

Results. During a 6-year period 195 NTDs were identified at a Western Cape Province tertiary hospital. These included 59 (30\%) cases of anencephaly, 28 (14\%) of encephalocele and 108 (55\%) of SB. The majority of NTDs (71\%) were detected prenatally, although SB was less commonly diagnosed prenatally than cranial defects ( $56 \%$ v. $88 \%$; $p<0.001)$. Of SB cases ascertained pre- or postnatally, $57 \%$ of patients were born alive and $50 \%$ discharged alive, but $72 \%$ of survivors had not been diagnosed prenatally. Women receiving prenatal diagnosis of any type of NTD before 24 weeks' gestation were nearly always offered TOP, and the majority accepted termination after non-directive counselling. For SB, later prenatal diagnosis was associated with much lower termination rates because the option was less often offered ( $51 \%$ v. $100 \% ; p<0.001)$, and perhaps less often accepted ( $57 \%$ v. $78 \% ; p=0.06)$. The estimated NTD birth prevalence for the referral area was 0.76 - 0.80 per 1000 live births, but perhaps up to 1.18 per 1000 when considering under-referral of lethal cranial lesions from rural areas. Conclusions. A substantial number of NTDs can be ascertained from a tertiary hospital environment if multiple data sources are used, even though adding data from the Perinatal Problem Identification Program for outlying health facilities increases detection of lethal defects. Hospital-based surveillance can be considered, especially for SB. Prenatal diagnosis was fairly common and pregnancy termination was often offered and accepted if detected before 24 weeks' gestation. A regional prenatal ultrasound programme, predominantly based in primary care but with ready access to a tertiary centre, can be quite effective, although limited or delayed access to prenatal diagnosis must be addressed.

S Afr Med J 2019;109(9):698-703. https://doi.org/10.7196/SAMJ.2019.v109i9.13863

Neural tube defects (NTDs) include anencephaly, encephalocele and spina bifida (SB). Anencephaly is a lethal condition, whereas encephalocele and SB, which may cause long-term disability, are a considerable burden to families and the healthcare system, ${ }^{[1,2]}$ and are relatively common birth defects. In South Africa (SA), studies give estimated population prevalence rates of 0.99 - 3.8 per 1000 live-born infants. ${ }^{[3-6]}$

Folic acid supplementation in the peri-conceptional period ${ }^{[7]}$ or fortification of staple foods ${ }^{[8]}$ has been shown to reduce the prevalence of NTDs. In SA, the introduction of folic acid fortification of maize and wheat products in 2003 was associated with a $31 \%$ reduction in NTD prevalence at sentinel sites, from 1.41 to $0.98 / 1000$ births. ${ }^{[9]}$ Another preventive option is prenatal diagnosis by ultrasound, with the choice of termination of pregnancy (TOP), which has become increasingly available in parts of SA. Despite these changes, there are minimal data on NTD prevalence after 2003.

Effective surveillance of NTDs and other birth defects is important for various reasons, e.g. to establish burden of disease, monitor trends in birth prevalence, assess impact of preventive measures and detect outbreaks. The last role is highlighted by the recent link between maternal Zika virus infection and microcephaly in offspring, ${ }^{[10]}$ and the possible link between maternal use of the antiretroviral drug dolutegravir and NTDs. ${ }^{[11]}$

The National Department of Health $(\mathrm{NDoH})$ has a populationbased birth defect surveillance system, which appears to be ineffective. ${ }^{[12]}$ Hospital-based surveillance is an easier alternative, but less optimal because of lack of a defined denominator. It therefore works best where it captures most of the population of interest in a geographical region. ${ }^{[13]}$ We anticipated this to be largely true in Western Cape Province, SA, for NTDs, or at least for SB, given that both prenatal diagnosis and paediatric management are centralised at tertiary hospitals with defined catchment areas. Furthermore, most NTDs are readily visible at birth. We therefore aimed to assess the detection of NTDs at a tertiary hospital using a range of prenatal, perinatal and postnatal data sources, and to estimate the impact of prenatal diagnosis and birth prevalence for the referral area.

\section{Methods}

The study was conducted at Tygerberg Academic Hospital (TAH), a tertiary public hospital in the Western Cape. It is a referral centre for 
approximately half of the province, including the eastern half of the Cape Town metropole (urban) and small towns and farms up to $300 \mathrm{~km}$ distant (rural).

The Western Cape DoH mandates that, as far as possible, all pregnant women have access to an ultrasound assessment for fetal anomalies if they initiate care before 24 weeks' gestation. In most cases, this is provided by sonographers at level 1 and 2 health facilities or mobile units. If a fetal anomaly is detected, the patient is referred to TAH for expert ultrasound assessment, non-directive genetic counselling and further management. This may include TOP, which is available for nearly all NTDs until 24 weeks' gestation, and thereafter only if the prognosis is considered to be very poor. If born outside TAH, live-born infants with NTDs from the same catchment area are similarly referred to TAH for neurosurgical management, unless treatment is considered futile.

Data regarding NTDs seen at TAH were collected retrospectively for 6 years in the post-fortification era $(2003,2004,2007,2009,2011$ and 2012), for which multiple data sources were available. NTDs were classified as: anencephaly (with or without $\mathrm{SB}$ ), encephalocele (with or without SB), and SB with intact cranium. Data were obtained from 5 sources to maximise case ascertainment: the Fetal Medicine Unit (FMU) database, stillbirth assessment records for TAH, register of the neonatal intensive care unit (NICU), Perinatal Problem Identification Program (PPIP) and registers of the emergency and neurosurgical theatres. All 5 data sources were available for all 6 years assessed.

The FMU database is a bespoke software system, Astraia (Astraia software gmbh, Germany), where clinical data, ultrasound findings and management plans are documented prospectively during actual antenatal visits, and used for clinical communication. The information in the database is therefore a prospective record of all NTD patients seen prenatally at TAH.

Permission was obtained to access the PPIP database for TAH, which collects data for perinatal deaths (stillbirths with birthweight $>500 \mathrm{~g}$, or deaths before 1 week of age) as part of a national surveillance system of perinatal deaths. The programme is championed by an interested staff member, and data are prospectively gathered and linked to a weekly morbidity and mortality meeting attended by obstetricians, neonatologists, medical geneticists and pathologists. It provides quite accurate and comprehensive information on all perinatal deaths of infants delivered at TAH.

Data on intrauterine deaths (IUDs) were obtained from the stillbirth assessment records of the Tygerberg Hospital Clinical Unit of Medical Genetics and Genetic Counselling. It is policy that all IUD fetuses at TAH be kept for inspection by a medical geneticist. Fetuses are also referred from other facilities in the metropolitan catchment area if the staff note an anomaly. The records contribute to PPIP data, but also include fetuses weighing $<500 \mathrm{~g}$ (often the result of a medical TOP).

Lastly, registers of the NICU and emergency and neurosurgical theatres were retrospectively reviewed for the entire study period. It was impossible to identify postnatally diagnosed cases, where patients were admitted to the general neonatal wards but did not undergo surgery, as this information is not routinely captured.

Cases originating from different sources were carefully crosschecked to exclude duplication.

In addition to the 6-year comparative review, the FMU database was used to assess the offer and uptake of TOP for a 10-year period (2003 - 2012), as it is nearly a complete representation of all prenatally diagnosed cases in the drainage area. The only exceptions could have been cases with unequivocal diagnosis of anencephaly at one of the two regional hospitals (although these are usually referred to TAH). Timing of TOP was considered 'late' if it occurred after 24 weeks of gestation, as TOP would then often involve a feticide procedure and policy therefore is more restrictive.

For all prenatally detected cases, information regarding TOP or fetal demise was obtained from Astraia or maternal records. Where available, hospital records of all live-born infants were reviewed to assess whether the child died in hospital or was discharged alive.

To estimate NTD prevalence in the referral area, denominator data for live births in all health facilities were obtained from the Western Cape DoH. These records include all births at healthcare facilities, or before arrival at the facility, and allowed separation between rural and urban births. Place of residence for cases of NTD (the numerator) was determined from hospital records. Due to changes in referral area over time, estimation of prevalence was only possible for 2011 - 2012.

Statistica 12.0 software (StatSoft, USA) was used to provide descriptive data and comparisons based on maternal and lesion characteristics. A univariate analysis of the data was conducted, with $p \leq 0.05$ considered statistically significant.

\section{Ethical approval}

Institutional Review Board approval for the study was granted by the Health Research Ethics Committee of Stellenbosch University (ref. no. S13/06/064). This included a waiver of informed consent for all participants. To maintain confidentiality of data, the study database was de-identified and password protected.

\section{Results}

During the 6 years that data from all 5 sources were available, a total of 195 cases of NTD were identified. These included 59 (30\%) cases of anencephaly, 28 (14\%) of encephalocele and 108 (55\%) of SB, of which 52, 25 and 61, respectively, had been detected prenatally (with an $8 \%$ incidence of twinning). Nine patients with anencephaly and 1 with encephalocele also had SB.

The number of cases identified per year was quite variable, but with no identifiable temporal trend (Fig. 1). The proportion detected prenatally varied from a low of 55\% in 2003 to a peak of $85 \%$ in 2009 . There was a non-significant trend towards improvement in prenatal detection over time $(r=0.56 ; p=0.25)$.

Across the 5 data sources a total of 340 NTD identifications was made, with significant overlap between the sources. Of the 195 cases, 29 (15\%) were identified by 3 sources, 87 (45\%) by 2 sources, and $79(41 \%)$ by a single data source. Fig. 2 shows the number of cases identified per source, and the number identified only by that source. The largest single contributor was the FMU database (138/195; 71\%). This also contributed the largest number of unique single-source identifications ( 46 cases; $24 \%$ of all cases; and $58 \%$ of cases identified by a single source), while the PPIP database contributed no unique cases (Fig. 2). Retrospectively collected data from the registers of the NICU and theatres contributed $47(24 \%)$ cases that were not otherwise identified.

The accuracy of ultrasound diagnosis in the FMU was assessed for 92 patients seen both prenatally and postnatally. Prenatal detection occurred in all 50 cases of confirmed cranial defects, and in 41 of 42 with SB (sensitivity $97.6 \%$; $95 \%$ confidence interval (CI) 86.6 - 99.9). All 41 prenatally detected cases were confirmed postnatally (positive predictive value 100\% (95\% CI 92.6 - 100). The single false-negative scan was of a patient first seen at 35 weeks' gestation with ventriculomegaly, and visualisation was affected by late gestation, supine fetal position and anhydramnios due to bilateral renal pathology.

Evidence of syndromic features or a specific underlying cause was found in $17 \%(34 / 195)$ of cases, including 17\% (10/59) of anencephaly, $43 \%(12 / 28)$ of encephalocele and $11 \%(12 / 108)$ of 


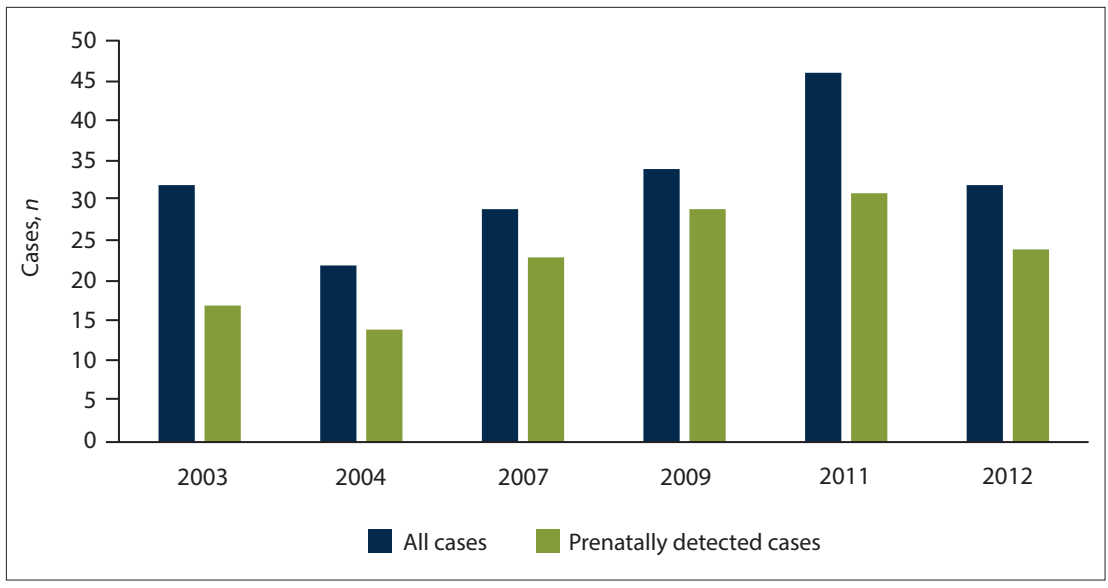

Fig. 1. Number of cases detected per year - total and prenatal.

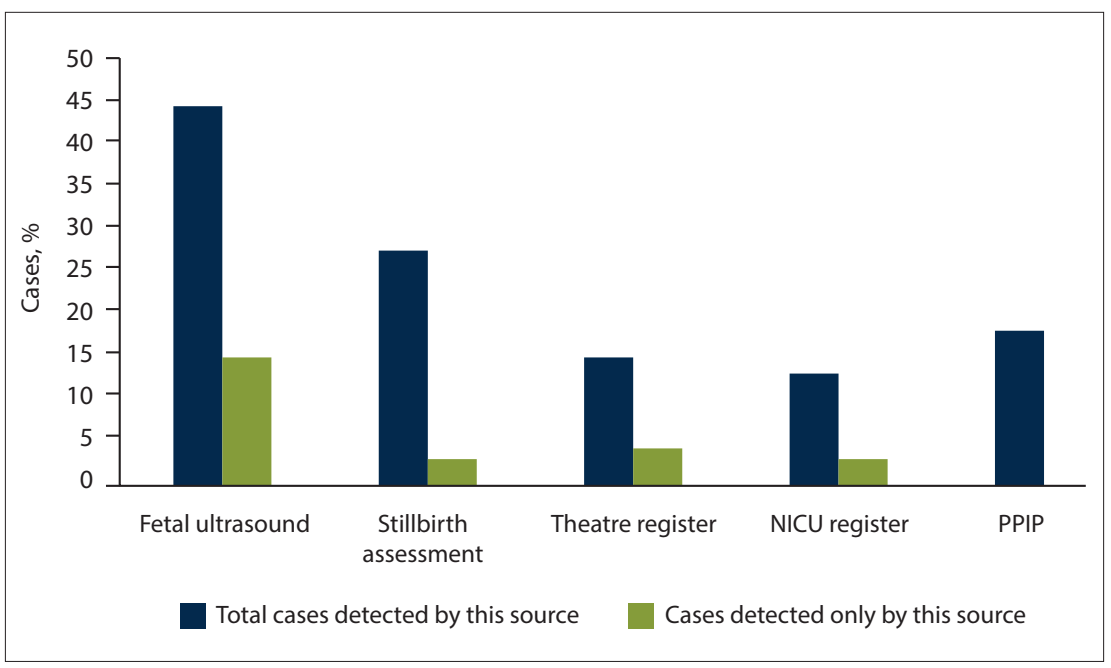

Fig. 2. Percentage of cases detected by each data source. (NICU = neonatal intensive care unit; PPIP= Perinatal Problem Identification Program.)

SB $(p<0.001)$. The most frequently detected causes were amniotic bands $(6 \% ; 11 / 195)$ and chromosomal abnormalities $(2 \% ; 4 / 195)$, while $6 \%(11 / 195)$ were assigned as having 'multiple congenital anomalies' due to the presence of anomalies in other organ systems, or multiple dysmorphic features.

Table 1 describes outcomes up to discharge (either dead or alive), which were known for $94 \%(184 / 195)$ of cases. Of the 184 NTD patients with known outcome, $41 \%$ (75/184) were born alive and 31\% (57/184) discharged alive. For SB, corresponding figures were 57\% (59/103) and 50\% (52/103), respectively. Of the 57 survivors who were discharged from hospital, 41 cases (72\%; 3 with encephalocele and 38 with SB) had not been detected prenatally. Of the 109 fetuses that were not born alive, TOP was done in 92 (84\%), and IUD occurred in 17 (16\%).

Of the 195 cases, 138 (71\%) were prenatally diagnosed, and 92 (47\%) received TOP. Both prenatal diagnosis and TOP occurred less frequently for SB than for cranial defects $(p<0.001)$.
Antenatal management decisions were assessed in detail, using the full 10-year (2003 - 2012) dataset of 229 prenatally detected cases. TOP was offered to $87 \%$ of women, including $99 \%$ with a diagnosis before 24 weeks' gestation - the one exception being an anencephaly with a healthy co-twin and $67 \%$ after 24 weeks' gestation (Table 2). Eighteen (9\%) of the 199 women who were offered TOP were lost to follow-up, but $78 \%$ of the remaining 181 accepted TOP (71\% overall TOP acceptance if all lost to follow-up cases would have opted against TOP). For all NTDs collectively, none of the following factors was associated with choice of TOP: urban or rural residence $(p=0.42)$, diagnosis in the first or second half of the study period ( $p=0.96$ ), age $\geq 37$ or $<37$ years $(p=0.49)$, sonographic appearance of syndromic features $(p=0.71)$, or diagnosis before or after 24 weeks' gestation $(p=0.35)$.

Uptake of TOP was high for all lesions before 24 weeks' gestation, but late TOP was less frequent for cases of SB than cranial lesions. Compared with $<24$ weeks, late TOP for SB was much less common (odds ratio (OR) 0.10 ; 95\% CI $0.04-0.26$; $p<0.001$ ), because TOP was much less frequently offered (51\% (23/45) v. 100\% (74/74); $p<0.001)$ and perhaps less often accepted $(57 \%(12 / 21)$ v. $78 \%(50 / 64) ; p=0.06)$.

The minimum birth prevalence of NTDs (cases per 1000 live births) in the TAH catchment area was estimated for 2011 and 2012. During this period, there were 76 cases of NTDs and 93609 live births. As at least 71 but probably 75 women resided in the drainage area, the minimum overall NTD birth prevalence estimate was 0.76/1 000 (95\% CI 0.59 - 0.96) and the maximum estimate $0.80 / 1000$ (95\% CI 0.63 - 1.00), similar for rural and urban areas.

Estimated birth prevalence for urban and rural areas differed significantly according to NTD type (Table 3). For cranial lesions, the estimated birth prevalence was higher in urban areas, but for SB it was twice as high in rural than urban areas $(0.47 / 1000$ (95\% CI 0.28 $0.750)$ v. $0.23 / 1000$ (95\% CI $0.12-0.40$ ); $p=0.05)$.

\section{Discussion}

During 6 years of pre- and postnatal case ascertainment, the study identified nearly 200 patients with NTDs first presenting to a tertiary hospital. This confirms that, while folic acid fortification may have had an effect, NTDs remain a substantial health problem.

Lebese et al. ${ }^{[12]}$ described cases reported to the NDoH birth defect surveillance system, and raised concerns about severe underascertainment of cases. A comparison with our study lends weight to this: 195 new cases of NTDs were identified in 6 years (average of 33 cases/year) for a single tertiary hospital with its catchment area, including $<5 \%$ of SA's population and births. By comparison, the $\mathrm{NDoH}$ surveillance system detected 787 cases for an overlapping 9-year period (87 cases/year) from SA as a whole. Our study suggests that data available at tertiary hospital level are underutilised.

All data sources contributed to case finding, with $41 \%$ of NTDs being identified only by a single source, and with unique single-source cases being contributed by 4 of the 5 sources (Fig. 2). This indicates that optimal ascertainment needs data from prenatal, perinatal and postnatal sources. This applies not only to a retrospective study such as ours, but also to prospective hospital-based surveillance, and to other birth defects, even more so to defects that are less readily amenable to pre- or perinatal detection than NTDs.

It is known that TOPs and early IUDs are very difficult to ascertain postnatally, ${ }^{[13]}$ 
Table 1. Final patient outcomes in the 6-year period with prenatal and postnatal case ascertainment

\begin{tabular}{llllll}
\hline & Total, $\boldsymbol{n}$ (\%) & Anencephaly, $\boldsymbol{n}$ (\%) & Encephalocele, $\boldsymbol{n}$ (\%) & Spina bifida, $\boldsymbol{n}$ (\%) & $\boldsymbol{p}$-value \\
\hline Patients, total & 195 & $59(30)$ & $28(14)$ & $108(55)$ & \\
Outcome known & $184 / 195(94)$ & $54 / 59(92)$ & $27 / 28(96)$ & $103 / 108(95)$ & 0.52 \\
Prenatally detected & $138 / 184(75)$ & $52 / 59(88)$ & $25 / 28(89)$ & $61 / 108(56)$ & $<0.001$ \\
Termination of pregnancy & $92 / 184(50)$ & $38 / 54(70)$ & $18 / 27(67)$ & $36 / 103(35)$ & $<0.001$ \\
Stillbirth & $17 / 184(9)$ & $8 / 54(15)$ & $1 / 27(4)$ & $8 / 103(8)$ & 0.2 \\
Live born & $75 / 184(41)$ & $8 / 54(15)$ & $8 / 27(30)$ & $59 / 103(57)$ & $<0.001$ \\
$\quad$ Proportion prenatally diagnosed & $28 / 75(37)$ & $7 / 8(88)$ & $5 / 8(63)$ & $16 / 59(27)$ & 0.001 \\
Discharged alive & $57 / 184(31)$ & $0 / 54(0)$ & $5 / 27(19)$ & $52 / 103(50)$ & $<0.001$ \\
$\quad$ As \% of live born & $57 / 75(76)$ & $0 / 8(0)$ & $5 / 8(63)$ & $52 / 59(88)$ & $<0.001$ \\
$\quad$ Proportion prenatally diagnosed & $16 / 57(28)$ & - & $2 / 5(40)$ & $14 / 52(27)$ & 0.6 \\
Cases of intact cranium only. & & & & &
\end{tabular}

Table 2. Offer and acceptance rates for pregnancy termination over a 10-year period

\begin{tabular}{|c|c|c|c|c|c|}
\hline & Total, $n(\%)$ & Anencephaly, $n(\%)$ & Encephalocele, $n(\%)$ & Spina bifida, $n(\%)$ & $p$-value \\
\hline Patients, total & 229 & 75 & 35 & 119 & \\
\hline TOP offered & $199 / 229(87)$ & $69 / 75(92)$ & $33 / 35(94)$ & $97 / 119(82)$ & 0.04 \\
\hline TOP decision known & $181 / 199(91)$ & $63 / 69(91)$ & $33 / 33(100)$ & $85 / 97(88)$ & 0.2 \\
\hline \multirow[t]{2}{*}{ TOP after offered ${ }^{*}$} & $142 / 181(78)$ & $53 / 63(84)$ & $27 / 33(82)$ & $62 / 85(73)$ & 0.2 \\
\hline & & & & & $0.09^{\dagger}$ \\
\hline Patients, $n$ (<24 weeks) & 140 & 46 & 20 & 74 & \\
\hline TOP offered & $139 / 140(99)$ & $45 / 46(98)$ & $20 / 20(100)$ & $74 / 74(100)$ & 0.5 \\
\hline TOP decision known & $126 / 139(91)$ & $42 / 45(93)$ & $20 / 20(100)$ & $64 / 74(87)$ & 0.2 \\
\hline TOP after offered ${ }^{*}$ & $102 / 126(81)$ & $35 / 42(83)$ & $17 / 20(85)$ & $50 / 64(78)$ & 0.8 \\
\hline Patients, $n$ ( $\geq 24$ weeks) & 89 & 29 & 15 & 45 & \\
\hline \multirow[t]{2}{*}{ TOP offered } & $60 / 89(67)$ & $24 / 29(82)$ & $13 / 15(87)$ & $23 / 45(51)$ & 0.004 \\
\hline & & & & & $<0.001^{\dagger}$ \\
\hline TOP decision known & $55 / 60(92)$ & $21 / 24(88)$ & $13 / 13(100)$ & $21 / 23(91)$ & 0.6 \\
\hline \multirow[t]{2}{*}{ TOP after offered ${ }^{*}$} & $40 / 55(73)$ & $18 / 21(86)$ & $10 / 13(77)$ & $12 / 21(57)$ & 0.1 \\
\hline & & & & & $0.02^{\dagger}$ \\
\hline Feticide & $18 / 40(45)$ & $0 / 18(0)$ & $8 / 10(80)$ & $10 / 11(91)$ & $<0.001$ \\
\hline
\end{tabular}

Table 3. Birth prevalence (NTD cases per 1000 live births) by type and place of residence

\begin{tabular}{|c|c|c|c|c|}
\hline & Combined urban and rural ${ }^{*}$ & Urban* & Rural $^{*}$ & $p$-value \\
\hline Live births, $n$ & 93609 & 55643 & 37966 & \\
\hline Anencephaly, $n$ & 27 & 21 & 6 & 0.05 \\
\hline Prevalence (95\% CI) & $0.29(0.19-0.42)$ & $0.38(0.23-0.58)$ & $0.16(0.06-0.34)$ & \\
\hline Encephalocele, $n$ & 13 & 12 & 1 & 0.02 \\
\hline Prevalence (95\% CI) & $0.14(0.07-0.24)$ & $0.22(0.11-0.38)$ & $0.03(0.0-0.15)$ & \\
\hline Spina bifida, ${ }^{\dagger} n$ & 31 & 13 & 18 & 0.05 \\
\hline Prevalence (95\% CI) & $0.33(0.22-0.47)$ & $0.23(0.12-0.4)$ & $0.47(0.28-0.75)$ & \\
\hline All NTDs, $n$ & 71 & 46 & 25 & 0.4 \\
\hline Prevalence (95\% CI) & $0.76(0.59-0.96)$ & $0.83(0.61-1.1)$ & $0.66(0.43-0.97)$ & \\
\hline
\end{tabular}

but use of prenatal data allowed us to identify many such cases, with prenatal cases accounting for $71 \%$ of all NTDs, and $24 \%$ of cases being recorded only prenatally (Fig. 1). Failure to include prenatal data would thus have caused considerable underestimation of the true NTD prevalence.

Stillbirth assessment records identified $23 \%$ of cases and the PPIP database $15 \%$. Most of these overlapped with prenatally detected cases, but where prenatal data are not available, other sources assume importance.

To assess detection at TAH, we also reviewed data from other health facilities in the catchment area participating in the PPIP and identified 83 cases -55 urban and 28 rural. Of these, 30 were detected at TAH (29 prenatally, but most also by TAH stillbirth and PPIP records). Therefore, the additional PPIP data sources may increase 
the total number of identifiable cases to as many as 248 . It should be noted that the additional PPIP data did not include information on type of NTD and most likely included mainly anencephaly, did not provide full coverage of the rural catchment area, and could not always be easily assessed for duplication with TAH data. Therefore, we restrict ourselves to a discussion of TAH data, except where specifically indicated otherwise.

Of cases detected at TAH, live births comprised only a fraction (41\% of all NTD and $57 \%$ of SB cases), with further attrition occurring by hospital discharge. Surveillance for NTDs that targets only live-born infants or patients attending outpatient clinics would therefore give a severe underestimate of the true birth prevalence.

Comparison of prenatal and postnatal sources shows that sonographic detection of NTDs in the FMU was very accurate, with all cranial defects and all but one case of SB being detected. The sensitivity for detection of SB was similar to that quoted for expert centres. ${ }^{[14]}$

The overall prenatal detection rate for NTDs, however, was only $71 \%$. This is lower than the $80-100 \%$ reported from countries with well-resourced fetal medical services, ${ }^{[15-17]}$ yet probably very high by SA's public health system standards, as the FMU at TAH has a wellestablished network of sonographers at primary and secondary care levels. This gives access to ultrasound screening for pregnant women who initiate antenatal care before 24 weeks' gestation throughout the TAH drainage area. Referral pathways to tertiary care are also well established.

Maternal records were not available for postnatal patients delivered elsewhere; hence, we were unable to determine why a prenatal diagnosis was not made. It is probably owing to a combination of lack of prenatal ultrasound screening (possibly due to late booking or staff-related omissions) or false-negative examinations (possibly due to lack of experience or excessive case load of sonographers, combined with high levels of obesity in the population). These reasons warrant further study and continued education of both staff and women to optimise use of the Western Cape policy that offers a detailed ultrasound scan for all women at $19-22$ weeks of gestation.

This study found a relatively high rate of TOP for prenatally detected NTDs $-70 \%$ for cranial lesions and $56 \%$ for SB. However, this represented only $35 \%$ of all SB cases - a discrepancy that relates in part to $29 \%$ not receiving prenatal diagnosis at their local facility.

Further points emerged from the 10-year prenatal data (Table 2). TOP decisions were unknown for $9 \%$ of NTD (12\% of SB) cases, which may cause over- or underestimation of the TOP rate. Before 24 weeks' gestation, the acceptance rate for TOP was $73-80 \%$ for all NTDs (depending on whether none or all cases with missing outcomes had TOP) with figures for SB being 68 - 81\%. Given that the counselling provided was non-directive, this suggests a high degree of acceptability of early TOP for NTDs.

Diagnosis of SB after 24 weeks' gestation reduced the proportion of patients who were offered TOP by half, and perhaps acceptance of TOP by $>25 \%$ (Table 2). A similar effect has been described in a developed country. ${ }^{[18]}$ The need to offer feticide with late TOP has practical and emotional implications for both staff and patients, and means that regional policy guidelines restrict late TOP to patients with specific poor prognostic features. The problem is magnified by $\sim 40 \%$ of prenatal SB diagnoses being late.

The estimated birth prevalence for NTDs in our study was $0.76-0.80$ per 1000 live births (anencephaly 0.29/1 000 (95\% CI 0.19 - 0.42) and SB 0.33/1 000 (95\% CI 0.22 - 0.47) ) (Table 3). Adding PPIP data from outlying institutions increased the estimated prevalence of NTDs to 0.94/1 000 (1.02/1 000 urban; 0.82/1 000 rural). These estimates can be compared only cautiously with those in previous studies, because of differences in study design and ascertainment. A prospective study of the effect of folic acid fortification found a post-fortification prevalence of NTDs of $0.98 / 1000$ births (anencephaly $0.37 / 1000$; SB 0.54/1 000) in a mixed urban and rural population, but in other regions of SA. ${ }^{[9]}$ The last published data from Cape Town were before the introduction of folic acid fortification, and gave an NTD prevalence of $0.95-1.05 / 1000$ live births for populations similar to ours. ${ }^{[3,19]}$

The proportions of cranial and spinal lesions originating from urban and rural areas in this study suggest some underestimation, which is corroborated by the detection of additional lethal cases in PPIP data from outlying facilities. Specifically, we suspect that there was under-ascertainment of severe cranial lesions from rural areas, both pre- and postnatally, because such cases are easily recognised and may not have been referred to a tertiary level hospital. It is likely that PPIP data from outlying institutions do not fully capture this effect. In contrast, we expect the SB data to be relatively accurate, because the condition is usually associated with live birth (in the absence of TOP), is easily visible at birth and requires urgent neonatal surgery at a tertiary hospital.

Studies from the pre-fortification era indicate a higher birth prevalence of NTDs in rural than urban SA populations. ${ }^{[3-6,19]}$ This accords with our finding that $\mathrm{SB}$ was twice as common in rural areas. If one assumes that our data for SB are accurate, and corrects the prevalence of cranial lesions accordingly, the true population prevalence of NTDs in the catchment area might be as high as 1.18/1 000 live births. Although folic acid fortification may have had some effect on the prevalence, NTDs certainly remain a significant problem. As with birth defects and non-communicable diseases more generally, it can be predicted that their contribution to the burden of disease will increase over time. ${ }^{[20]}$

Taken together, the findings suggest that we identified a substantial proportion of NTD patients, especially with SB, born in the TAH catchment area. This is perhaps surprising, given that ours was a retrospective and hospital-based study, and is indicative of good record-keeping at the hospital. The data also suggest that hospitalbased prospective surveillance, even if limited to tertiary hospitals, should be useful if care is taken with data collection and multiple data sources are used. Depending on the quality of PPIP data at outlying health facilities, this may add to the completeness of the picture obtained at tertiary level. This approach is less resource and labour intensive than population-based surveillance.

\section{Study strengths and limitations}

The retrospective nature of the study is a limitation. However, the prenatal and stillbirth data for TAH are quite accurate, as these were collected prospectively by the fetal medicine and medical genetics groups. The postnatal data from the NICU and theatre registers were retrospectively collected and may be less complete, but nonetheless added significantly to the number of cases identified. This dataset is the optimal case ascertainment that can be achieved from routinely collected information at the hospital.

The use of multiple data sources strengthened our study, and the majority of cases were corroborated by more than one source, with the risk of accidentally inflating the number of cases being minimised by careful cross-checking of the different sources. In particular, prenatal ascertainment and evidence for prenatal diagnosis were accurate, which was a strength, because TOP and early IUDs (contributing $56 \%$ of cases) are otherwise particularly difficult to ascertain postnatally. ${ }^{[13]}$

As the study was based in a tertiary hospital, caution should be observed in extrapolating the findings to the population as a 
whole. Review of PPIP data from outlying facilities suggests that incorporating this information may increase detection by $>20 \%$. Another potential source of data loss is the provision of treatment, either prenatally or postnatally, at tertiary facilities outside the referral area. However, the existing referral pathways and experience of colleagues in the fetal medicine and neurosurgical services suggest that this is uncommon.

The relative prevalence rates for the different NTDs helped to identify potential gaps in the data. These may be biased towards urban cases and less severe anomalies, as cases considered to be lethal (whether diagnosed prenatally or at birth) were probably not always referred to TAH, especially not from remote rural areas. Lack of identification of lethal cases from rural areas also means that the proportion of NTDs detected prenatally is somewhat overestimated.

\section{Conclusions}

NTDs are important birth defects that are key targets for both surveillance and prenatal diagnosis. We used 5 data sources to identify NTDs at a tertiary hospital over 6 years, identifying a substantial number of cases.

Information about facility-based surveillance is important because it is simpler than a population-based approach. Facility-based surveillance is becoming more feasible in the Western Cape for two main reasons: (i) the patient number is now a unique identifier issued at birth and used in all facilities across the province; and (ii) numerous clinical databases using the patient number are being linked. Our findings show that much data about NTDs are readily available at tertiary hospitals, even though these may not currently be collected as routine data. We also show the need to use multiple data sources covering the prenatal, perinatal and postnatal periods, and show the relative importance of individual sources in our setting. The addition of PPIP data from other facilities in the catchment area increases detection, but collection of data needs to be better harmonised between sites.

We estimated the minimum NTD birth prevalence for the catchment area population to be $0.76-0.80 / 1000$ live births using TAH data, and up to $0.94 / 1000$ if including lethal defects from all facilities that collect PPIP data in the catchment area. Both estimates are probably lower than the true prevalence, given the differential case ascertainment from rural and urban areas for SB compared with anencephaly or (large) encephalocele. Based on our data, it remains possible that the prevalence has not substantially decreased with the introduction of folic acid fortification of food.

Of SB cases ascertained pre- or postnatally, $57 \%$ of infants were born alive and $50 \%$ discharged alive but nearly three-quarters of survivors had not been diagnosed prenatally. Women who were diagnosed prenatally before 24 weeks' gestation were nearly always offered TOP and, for all types of NTD, the majority opted for the procedure after non-directive counselling. Later prenatal diagnosis was associated with much lower TOP rates for SB, because the option was much less often offered and perhaps also less often accepted.

In this region, a relatively high proportion of NTDs are diagnosed prenatally. We show that a regional prenatal ultrasound programme, predominantly based in primary care but with ready access to a tertiary centre, can be quite effective. There is a need to optimise access to timeous detailed fetal anomaly scanning of high quality.

\section{Declaration. None.}

Acknowledgements. We would like to acknowledge Prof. G de Jong for collecting much of the stillbirth data over many years, Dr S Gebhardt and the Perinatal Problem Identification Program for providing data on NTDs, and the Western Cape Department of Health for information on the number of live births in the referral area.

Author contributions. All authors contributed to the study design, data analysis and manuscript writing. Data collection was done primarily by EK. Funding. None.

Conflicts of interest. None.

Mitchell LE, Adzick NS, Melchionne J, Pasquariello PS, Sutton LN, Whitehead AS. Spina bifida. Lancet 2004;364(9448):1885-1895. https://doi.org/10.1016/S0140-6736(04)17445-X

2. Oakeshott P, Hunt GM, Poulton A, Reid F. Expectation of life and unexpected death in open spina bifida: A 40-year complete, non-selective, longitudinal cohort study. Dev Med Child Neurol 2010;52(8):749-753. https://doi.org/10.1111/j.1469-8749.2009.03543.x

3. Buccimazza SS, Molteno CD, Dunne TT, Viljoen DL. Prevalence of neural tube defects in Cape Town, South Africa. Teratology 1994;50(3):194-199. https://doi.org/10.1002/tera.1420500304

. Delport AD, Christianson AL, van den Berg HJ, Wolmarans L, Gericke GS. Congenital anomalies in black South African liveborn neonates at an urban academic hospital. S Afr Med J 1995;85(1):11-15. 5cayiyana D. Neural tube defects among rural blacks in a Transkei district. S Afr Med J 1986;69(10):618-620

6. Venter PA, Christianson AL, Hutamo CM, Makhura MP, Gericke GS. Congenital anomalies in rural black South African neonates - a silent epidemic? S Afr Med J 1995;85(1):15-20.

7. Czeizel AE, Dudás I. Prevention of the first occurrence of neural-tube defects by peri7. Czeizel AE, Dudás I. Prevention of the first occurrence of neural-tube defects by peri-
conceptional vitamin supplementation. N Engl J Med 1992;327(26):1832-1835. https://doi.org/10.1056/ conceptional vitamin sup

8. Castillo-Lancellotti C, Tur JA, Uauy R. Impact of folic acid fortification of flour on neural tube 8. Castillo-Lancellotti C, Tur JA, Uauy R. Impact of folic acid fortification of flour on neural tube
defects: A systematic review. Public Health Nutr 2013;16(5):901-911. https://doi.org/10.1017/ S1368980012003576

9. Sayed AR, Bourne D, Pattinson R, Nixon J, Henderson B. Decline in the prevalence of neural tube defects following folic acid fortification and its cost-benefit in South Africa. Birth Defects Res A Clin Mol Teratol 2008;82(4):211-216. https://doi.org/10.1002/bdra.20442

10. Schuler-Faccini L, Ribeiro EM, Feitosa IML, et al. Possible association between zika virus and microcephaly - Brazil 2015. MMWR Morb Mortal Wkly Rep 2016;65(3):59-62. https://doi. org/10.15585/mmwr.mm6503e2

11. Zash R, Makhema J, Shapiro RL. Neural-tube defects with dolutegravir treatment from the time of conception. N Engl J Med 2018;379(10):979-981. https://doi.org/10.1056/NEJMc1807653

12. Lebese V, Aldous C, Malherbe HL. South African congenital disorders data, 2006 - 2014. S Afr Med J 2016;106(10):992-995. https://doi.org/10.7196/SAMJ.2016.v106i10.11314

13. World Health Organization/Centers for Disease Control and Prevention/International Clearinghouse . World Health Organization/Centers for Disease Control and Prevention/International Clearinghouse
for Birth Defects Surveillance and Research. Birth Defects Surveillance: A Manual for Programme for Birth Defects Surveillance an
Managers. Geneva: WHO, 2014.

14. Nicolaides KH, Campbell S, Gabbe SG, Guidetti R. Ultasound screening for spina bifida: Cranial and cerebellar signs. Lancet 1986;2(8498):72-74. https://doi.org/10.1016/S0140-6736(86)91610-7

15. Boyd PA, Devigan C, Khoshnood B, Loane M, Garne E, Dolk H. Survey of prenatal screening policies in Europe for structural malformations and chromosome anomalies, and their impact on detection and termination rates for neural tube defects and Down's syndrome. Br J Obstet Gynaecol 2008;115(6):689-696. https://doi.org/10.1111/j.1471-0528.2008.01700.x

16. Salvador J, Arigita M, Carreras E, Lladonosa A, Borrell A. Evolution of prenatal detection of neural tube defects in the pregnant population of the city of Barcelona from 1992 to 2006. Prenat Diagn 2011;31(12):1184-1188. https://doi.org/10.1002/pd.2863

17. Timbolschi D, Schaefer E, Monga B, et al. Neural tube defects: The experience of the registry of congenital malformations of Alsace, France, 1995 - 2009. Fetal Diagn Ther 2015;37(1):6-17. https:// doi.org $/ 10.1159 / 000362663$

18. Bodin CR, Rasmussen MM, Tabor A, et al. Ultrasound in prenatal diagnostics and its impact on Bodin CR, Rasmussen MM, Tabor A, et al. Ultrasound in prenatal diagnostics and its impact on
the epidemiology of spina bifida in a national cohort from Denmark with a comparison to Sweden. the epidemiology of spina bifida in a national cohort from Denmark with
Biomed Res Int 2018:2018:9203985. https://doi.org/10.1155/2018/9203985

19. Buccimazza SS. Prevalence of neural tube defects and the outcome of myelomeningocoele in Cape Town. PhD thesis. Cape Town: University of Cape Town, 1995.

20. Malherbe HL, Christianson AL, Aldous C. Need for services for the care and prevention of congenital disorders in South Africa as the country's epidemiological transition evolves. S Afr Med J 2015;105(3):186-188. https://doi.org/10.7196/SAMJ.9136

Accepted 18 March 2019 